\title{
Atomistic theory of dark excitons in self-assembled quantum dots of reduced symmetry
}

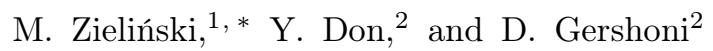 \\ ${ }^{1}$ Institute of Physics, Faculty of Physics, Astronomy and Informatics, \\ Nicolaus Copernicus University, Grudziadzka 5, 87-100 Toruń, Poland \\ ${ }^{2}$ The Physics Department and the Solid State Institute, \\ Technion-Israel Institute of Technology, Haifa, 32000, Israel
}

\begin{abstract}
We use an atomistic model to consider the effect of shape symmetry breaking on the optical properties of self-assembled InAs/GaAs quantum dots. In particular, we investigate the energy level structure and optical activity of the lowest energy excitons in these nanostructures. We compare between quantum dots with two-fold rotational and two reflections $\left(C_{2 v}\right)$ symmetry and quantum dots in which this symmetry was reduced to one reflection only $\left(C_{s}\right)$ by introducing a facet between the quantum dots and the host material. We show that the symmetry reduction mostly affects the optical activity of the dark exciton. While in symmetric quantum dots, one of the dark exciton eigenstates has a small dipole moment polarized along the symmetry axis (growth direction) of the quantum dot, in non-symmetric ones, the two dark excitons' dipole moments are predominantly cross-linearly polarized perpendicular to the growth direction and reveal pronounced polarization anisotropy. Our model calculations agree quantitatively with recently obtained experimental data.
\end{abstract}

PACS numbers: 78.67.Hc, 73.21.La, 78.55.Cr

Keywords: single quantum dot; micro-luminescence;

\section{INTRODUCTION}

Confined excitons in single semiconductor quantum dots (QDs) have been a subject of extensive studies since they play a central role in many schemes which lead towards applications in quantum optics and future quantum technologies. $\stackrel{1}{=}$ If a $\mathrm{QD}$ maintains two-fold rotational symmetry (such as $C_{2 v}$ ), its lowest-energy excitonic eigenstates can be divided into two characteristic doublets: the lowest energy doublet which is mostly optically inactive - the dark exciton (DE) - and the higher energy doublet which constitutes the fundamental optical excitations of the QD - the bright exciton, ${ }^{2.3}$ Bright excitons (BEs) have been thoroughly studied both experimentally and theoretically due to their obvious use in applications based on single photon sources, $\stackrel{4-6}{=} \sin -$ gle photon detectors, $\stackrel{7-9}{\underline{\underline{9}}}$ entangled photon sources $\underline{\underline{10}} \underline{-12}$ and photon-spin entanglement $\underline{13-15}$ On the contrary, relatively little is known about the nature of the dark excitons since their optical inactivity renders them quite difficult to access experimentally. Recently, however, it was demonstrated that QD-confined DEs, despite their weak optical activity in emission, can be efficiently accessed by optical absorption and by charge injection, 16,17 In this way, it was demonstrated that the DE actually forms a long lived two-level system (qubit) $\underline{16}$ with a long coher-

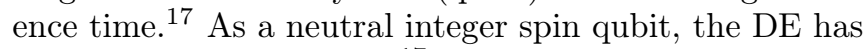
some obvious advantages 17 over the more conventional single carrier spin-based qubits $\stackrel{18-23}{-2}$ For these and other reasons, there is an increasing scientific interest in $\mathrm{DE}$ studies, $16,17,24$

From general theoretical considerations based on group theory, one expects that in symmetric QDs, the BE eigenstates have cross-linearly in-plane polarized dipole moments ( $\hat{x}$ and $\hat{y}$ directions, respectively), one of the $\mathrm{DE}$ eigenstates is completely dark (i.e. its dipole moment vanishes), and the other DE eigenstate may have a nonvanishing dipole moment, polarized along the QD symmetry axis ( $\hat{z}$ direction) $\underline{\underline{2}, 25,26}$ In order to obtain quantitative information about the magnitude of these dipole moments, however, more detailed theoretical modeling based on either effective mass approximations ${ }^{2,27,28}$ or atomistic calculations $25,26,29-31$ are required. Comparison with experimental studies $3,17,24,32,33$ are at times not easily obtained due to the lack of information regarding the actual size, composition, structure, and strain of an experimentally measured QD. In addition, the usually very weak optical activity of the DE is yet another obstacle to obtaining accurate experimental data. This obstacle can be partially removed by applying an external magnetic field perpendicular to the QD symmetry axis (Voigt geometry). Such a perturbation mixes between the $\mathrm{BE}$ and DE eigenstates, such that the mixed DE eigenstate gains a dipole moment and becomes optically active with in-plane polarized dipole moments, like those of the BE eigenstates. Extrapolations to zero magnetic field may yield information about the bare DE eigenstates as well. $, 17,24,32,33$

Modeling and structural simulations show that the magnitude of the dipole moment of the DE strongly depends on the actual shape of the QD and on its symmetry. For symmetric InAs/GaAs self-assembled QDs, it is generally accepted that the $\hat{z}$-polarized optical activity of the $\mathrm{DE}$ is due to hole subband mixing,2,24,25,31,34 Previous experimental studies which reveal residual unexplained in-plane optical activity of dark excitons attributed it to the reduced symmetry (lower than $C_{2 v}$ ) of the $\mathrm{QD} !^{3}$ Bayer et $a l^{\frac{3}{3}}$ suggested that the reduction in symmetry, like the in-plane external magnetic field, mixes between the $\mathrm{BE}$ and $\mathrm{DE}$ eigenstates, causing the later to become 
optically active.

In this paper, we used an atomistic model to realistically consider confined excitons in InGaAs/GaAs selfassembled semiconductor QDs of various shapes which maintain $C_{2 v}$ symmetry. We then introduce a structural perturbation in the QD shape which reduces the symmetry to $C_{s}$. We show indeed that such a perturbation is sufficient to trigger in-plane polarized optical activity of the DE eigenstates. We establish this way that while the in-plane (out-of-plane polarized) emission from the DE eigenstates can indeed be attributed to hole subband mixing, the out-of-plane (in-plane polarized) DE dipole moment is a very sensitive probe for the QD deviation from symmetry, and it mainly results from DE-BE mixing.

The use of an atomistic model is essential for an accurate, realistic description of the QD structure while maintaining the crystal lattice symmetry and keeping atomic scale details of the composition and strain distribution. We utilize empirical tight-binding theory for single particle states with an $s p^{3} d^{5} s^{*}$ orbitals, $\frac{35}{2}$ nearest-neighbor coupling, and spin-orbit and strain effects included 36 Relaxation of strain is accounted for via atomistic valence force field theory ${ }^{37,38}$ Piezoelectricity is included as well using both first- and second-order terms ${ }^{39}$ Exciton states are calculated using configuration-interaction treatment, which includes all possible determinants constructed from the 12 lowest-energy electron and 12 lowest-energy hole states (including spin) $\stackrel{40}{*}$ Finally, the optical spectrum is obtained by calculating the photoluminescence (PL) intensity due to the recombination of one electronhole pair in a given exciton state using Fermi's golden rule $\stackrel{30}{=}$

\section{HIGH-SYMMETRY QUANTUM DOTS}

Fig.11(a) and (c) show the geometry of two lens shaped QDs with the same circular base radius $(18 \mathrm{~nm})$ but different heights: $2.4 \mathrm{~nm}$ and $4.8 \mathrm{~nm}$, respectively. In Fig. 1 (b) and (d) we present the calculated excitonic emission spectra for these two symmetrical InAs/GaAs QDs. The QDs are located on a $0.6 \mathrm{~nm}$ (one lattice constant) thick InAs wetting layer. The overall QDs symmetry $\left(C_{2 v}\right)$ results from the combined zinc-blende lattice symmetry and the QD cylindrically symmetric lens shape. ${ }^{41,42}$ In effect, despite a nominally cylindrical base, the bright and dark excitonic doublets are non-degenerate, $, 42,43$ with pronounced bright exciton (BE) and dark exciton (DE) splitting. The bright exciton lines are polarized along non-equivalent [110] and [110] crystal axes, with small (3\% to $4 \%$ ) polarization anisotropy. In both QDs, a fraction of a meV below the lower energy BE spectral line, a weak emission line that originates from radiative recombination of the DE is observed. The visible DE line is polarized along the growth axis ([001]) of the QD, and the intensity of this line is about seven [six] orders of magnitude weaker than that of the BE in the short (Fig. 1 (a))
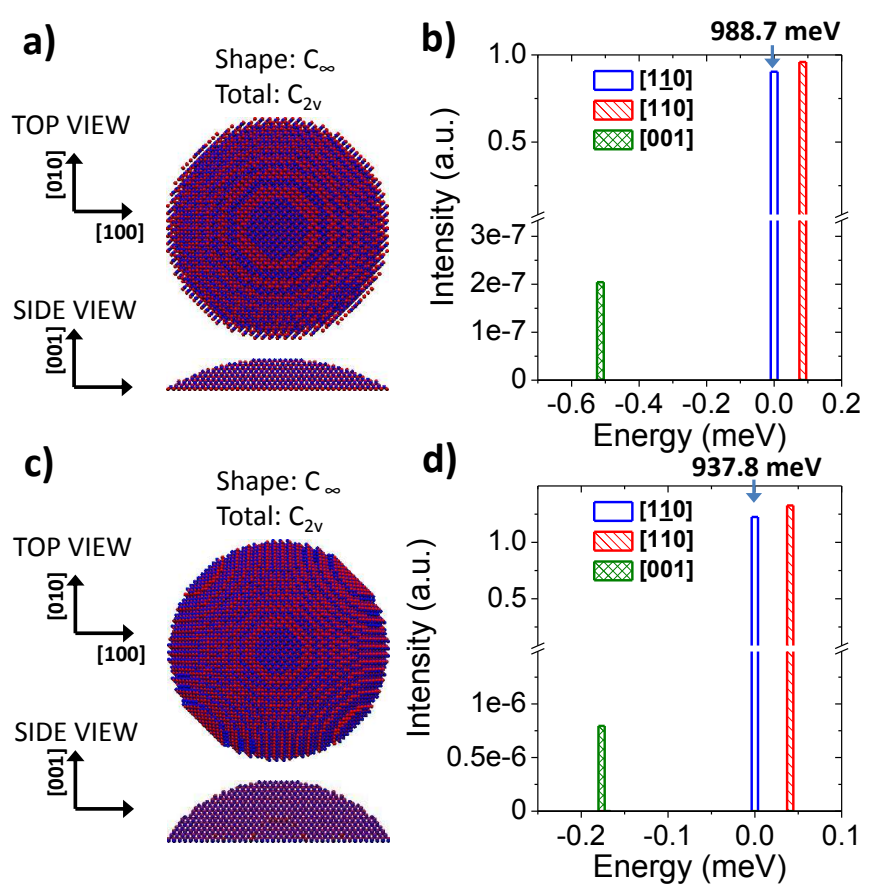

FIG. 1. (Color online) Schematic description of InAs/GaAs $C_{2 v}$ lens-shaped QD of $18 \mathrm{~nm}$ circular base radius and 2.4 $\mathrm{nm}$ (a) and $4.8 \mathrm{~nm}$ (c) height. Indium (Arsenide) atoms are shown as blue (red) spheres. Wetting layer atoms, as well as surrounding GaAs matrix atoms, are not shown. The calculated excitonic spectrum for the QDs in (a) and (c) are displayed in (b) and (d) respectively.

[tall (Fig. 1] (c))] QD. For both QDs, the lowest energy DE eigenstate remains completely optically inactive, and therefore, it is not shown in Fig. [1.

We note that we also studied numerous $C_{2 v}$ QDs with base shapes varying from ellipsoidal to a square base of a truncated pyramid (not shown) ${ }^{44}$ In these cases, the $z$-polarized dark exciton line can reach (truncated pyramid) a substantial fraction $\left(\approx 10^{-3}\right)$ of the bright exciton magnitude, i.e. three to four orders of magnitude stronger that of the cylindrically symmetric lens-shaped QDs. This increase of dark exciton activity can be attributed to the increased hole subband mixing. ${ }^{31}$ Nevertheless, the emission from the visible DE eigenstate of $C_{2 v}$ QDs is, as expected from symmetry considerations, always polarized along the QD growth axis.

\section{LOW-SYMMETRY QUANTUM DOTS}

In reality, ideally symmetrized systems of macroscopic scale are extremely rare. Recent theoretical studies of epitaxial growth of strained heterostructures ${ }^{45}$ concluded that self-assembled QDs can actually grow highly asymmetrically, largely deviating from any rotational symmetry. Realistic self-assembled QDs thus have symmetries which can deviate quite substantially from the idealized 
shapes of circular or ellipsoidal lenses. In order to methodically study the effects of the symmetry reduction on the optical properties of the QD, we introduced an inclined planar facet between the QD and the covering host material. In Fig. 2 (a) and (c), we present schematic depictions of two modified lens-shaped QDs, where the symmetry has been reduced by truncating several atomic layers, as depicted in Fig. 2] Fig. 2 (a) and (c) describe the structural modifications made to the symmetrical QDs in Fig. 1 (a) and (c), respectively, while the calculated excitonic spectra for these QDs is presented in Fig. 2 (b) and (d).

The overall QDs symmetry (structure and lattice) is now $C_{s}$ (reflection about one perpendicular plane). The choice of this shape-symmetry breaking mechanism is not intended to reproduce reality, but rather to introduce a certain well-controlled perturbation to otherwise perfectly circularly symmetric lens-shaped QDs. The truncation removes only $2 \%$ ( $8 \%$ in (c)) of the QD atoms, replacing them by the host material atoms. Therefore, the change in the BE emission energy of $5 \mathrm{meV}$ (3 meV in (d)) is rather small. Likewise, the BE emission intensities differ by no more than $3 \%$ (6\%) from those of the perfect lens in Fig. 1 (a) (Fig. 1 (c)). Both BE lines are in-plane polarized along the [110] and [110] crystallographic directions. Consistently, the BE-DE energy difference of $0.57 \mathrm{meV}(0.37 \mathrm{meV})$ is comparable with the $0.515 \mathrm{meV}$ $(0.315 \mathrm{meV})$ of the symmetrical QD. The BE fine structure splitting (FSS) is $48 \mu \mathrm{eV}(28 \mu \mathrm{eV})$, notably different from the $85 \mu \mathrm{eV}(41 \mu \mathrm{eV})$ observed for the symmetrical lens-shaped QD. This should not come as a surprise since the BE FSS is very sensitive to the QD structural

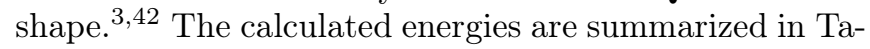
ble I.

Yet the most notable difference in the spectra from the asymmetric QDs is that the DE reveals in-plane linearly polarized emission. The intensity of the much brighter DE spectral line reaches $10^{-5}$ and $5 \times 10^{-4}$ of the $\mathrm{BE}$ emission intensity for the QD in (a) and in (c), respectively. The polarization directions follow those of the BEs, with the brighter one polarized like the lower energy BE line along the [1이 crystallographic direction. Apparently, both DE eigenstates gain some in-plane polarized oscillator strength, which is larger than their out of plane polarizations. The spectrum presented in Fig. 2 (d), in which the dipole moment of the much brighter DE eigenstate is about $1 / 1500$ that of the BEs, is in quantitative agreement with recently measured experimental results. 17

In the following, we discuss in more detail the effect of structural symmetry reduction on the exciton emission spectrum. We note first that, in atomistic calculations, due to the inclusion of the spin-orbit interactions on every atom and the presence of the underlying crystal lattice, neither the spin nor the orbital angular momentum are good quantum numbers, even for a perfectly circularly symmetric lens-shaped QD. Therefore, neither the calculated electron nor the hole ground states are eigen-
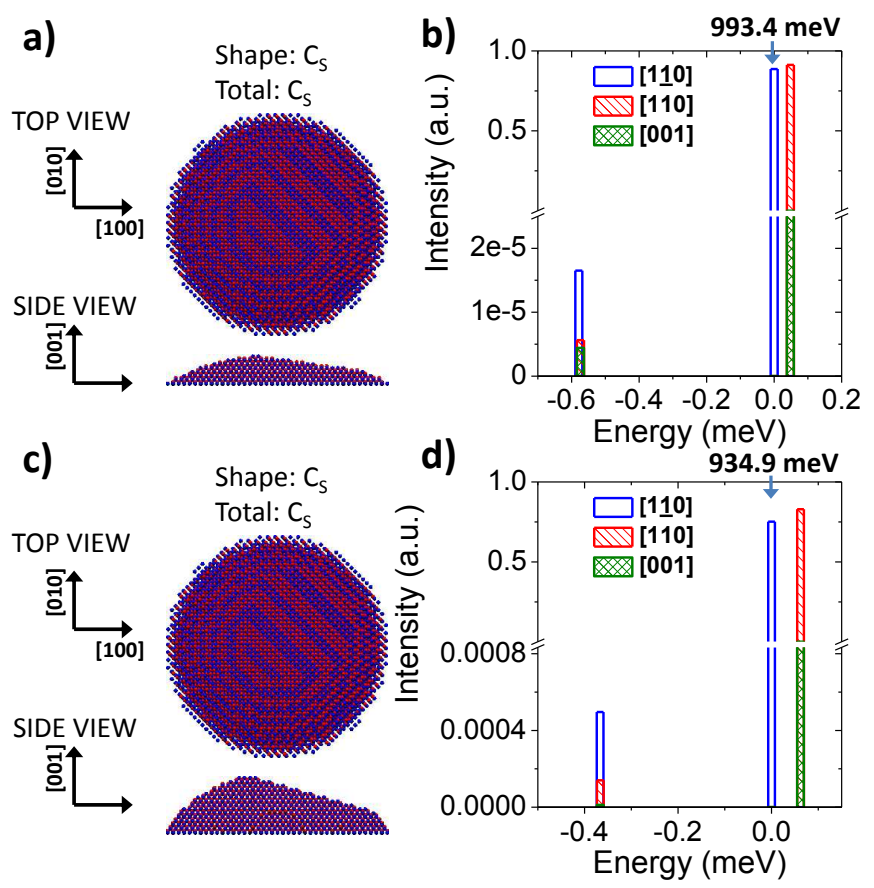

FIG. 2. (Color online) (a) [(c)] Schematic description of the lens-shaped QD in Fig. 1(a) [Fig. 1(c)] with a truncated planar facet. The calculated excitonic spectrum for the QDs in (a) and (c) are displayed in (b) and (d) respectively.

functions of the spin or the total angular momentum operators. Yet, in the absence of time reversal breaking interactions such as an external magnetic field, both the electron and the hole states are doubly (Kramers') degenerate. We label these four states as $e_{1}, e_{2}$ and $h_{1}, h_{2}$, respectively. The exciton energies are then calculated by including the Coulomb interaction between the electron and hole ground states. The direct Coulomb attraction reduces the energy of the exciton, while the exchange interactions remove the degeneracy between the four possible exciton eigenfunctions $\stackrel{2,3,27}{2}$

Using the exciton states $\left(|\mathrm{BE} 1\rangle=\left|e_{2} h_{2}\right\rangle,|\mathrm{BE} 2\rangle=\right.$ $\left.\left|e_{1} h_{1}\right\rangle,|\mathrm{DE} 1\rangle=\left|e_{1} h_{2}\right\rangle,|\mathrm{DE} 2\rangle=\left|e_{2} h_{1}\right\rangle\right)$ as a basis, the following exchange Hamiltonian is obtained for a system with $C_{2 v}$ symmetry. This general shape of the exchange Hamiltonian is anticipated from basic symmetry considerations ${ }_{2,3,27}^{2}$ We use our atomistic model and CI calculations to extract the matrix elements for this Hamiltonian,

$$
\mathcal{H}_{C_{2 v}}=\frac{1}{2}\left[\begin{array}{cccc}
\Delta_{0} & \mathrm{e}^{-\mathrm{i} \pi / 2} \Delta_{1} & 0 & 0 \\
\mathrm{e}^{\mathrm{i} \pi / 2} \Delta_{1} & \Delta_{0} & 0 & 0 \\
0 & 0 & -\Delta_{0} & \Delta_{2} \\
0 & 0 & \Delta_{2} & -\Delta_{0}
\end{array}\right]
$$

where $\Delta_{1}$ and $\Delta_{2}$ describe the DE and BE energy splitting, respectively, and $\Delta_{0}$ corresponds to the energy difference between the DE and the BE exciton doublets. The phase factors for the interaction between the $\mathrm{BE}$ configurations $|B E 1\rangle$ and $|B E 2\rangle$ are chosen so that their dipole moments are polarized parallel and perpendicu- 
lar to the [110] crystallographic direction, and $\Delta_{1}$ and $\Delta_{2}$ remain real numbers ${ }^{2,29}$ Normally, and in particular for nanosystems with a non-circularly symmetric base, the excitonic basis should be extended to include higher than ground electron and hole states, yielding a higher-dimensional Hamiltonian. $\underline{\underline{43}}$ Nevertheless, the $4 \times 4$ Hamiltonian qualitatively captures most of the physical properties of the QD-confined exciton fine structure. In the results reported here, the actual corrections to the calculated excitonic eigenenergies are rather small.

As seen from the Hamiltonian for the symmetric QD (Eq. 1), the DE and BE subspaces do not mix. The weak optical activity of one of the DE eigenstates is due to small, non-zero $\hat{z}$-polarized dipole matrix elements $\left|\left\langle e_{1}|\hat{z}| h_{2}\right\rangle\right|=\left|\left\langle e_{2}|\hat{z}| h_{1}\right\rangle\right|$, attributed predominantly to light-hole/heavy-hole mixing. These contributions interfere constructively (destructively) for the upper (lower) energy DE eigenstate. The in-plane polarized $(\hat{x}$ and $\hat{y})$ $\mathrm{DE}$ dipole moments for the QD of $C_{2 v}$ symmetry vanish: $\left|\left\langle e_{1}|\hat{x}, \hat{y}| h_{2}\right\rangle\right|=\left|\left\langle e_{2}|\hat{x}, \hat{y}| h_{1}\right\rangle\right|=0$.

The Hamiltonian (Eq. 10 is expressed in the basis of the four excitonic states calculated using the atomistic, tight-binding model. In the atomistic calculation, neither single particle states nor the excitonic configurations are eigenfunctions of the total angular momentum. In the literature,,$\frac{2.3}{.}$ however, the electron-hole exchange Hamiltonian is customarily expanded in the basis of the excitons with total angular momentum projections on the QD symmetry axis: $|+1\rangle,|-1\rangle,|+2\rangle,|-2\rangle$. In this basis, the states with $M= \pm 2$ cannot couple to light and they are termed DEs. The only optically active states are $M= \pm 1$ and they are termed BEs. Since, as discussed above, the the $4 \times 4$ Hamiltonian in both cases has exactly the same formal structure, one can readily make a one-to-one correspondence between the basis elements of both models and use the atomistic model to calculate the parameters of the Hamiltonian in the angular momentum representation.

For the QDs with lower symmetry $\left(C_{s}\right)$, the in-plane dipole moments do not vanish, i.e. $\left|\left\langle e_{1}|\hat{x}, \hat{y}| h_{2}\right\rangle\right| \neq 0$, allowing, in principle, DE emission (and absorption) along the growth direction of the QD. Depending on the deviation from symmetry, the magnitude of $\left|\left\langle e_{1}|\hat{x}| h_{2}\right\rangle\right|$ can reach a substantial fraction (up to $3 \%$ ) of that of the BE, $\left|\left\langle e_{1}|\hat{x}| h_{1}\right\rangle\right|$.

The electron-hole exchange Hamiltonian for the lowsymmetry QD $\left(C_{s}\right)$, as calculated by the atomistic CI model, is

$\mathcal{H}_{C_{s}}=\frac{1}{2}\left[\begin{array}{cccc}\Delta_{0} & \mathrm{e}^{-\mathrm{i} \pi / 2} \Delta_{1} & \mathrm{e}^{-\mathrm{i} \pi / 4} \Delta_{11} & \mathrm{e}^{-\mathrm{i} \pi / 4} \Delta_{12} \\ \mathrm{e}^{\mathrm{i} \pi / 2} \Delta_{1} & \Delta_{0} & -\mathrm{e}^{\mathrm{i} \pi / 4} \Delta_{12} & -\mathrm{e}^{\mathrm{i} \pi / 4} \Delta_{11} \\ \mathrm{e}^{\mathrm{i} \pi / 4} \Delta_{11} & -\mathrm{e}^{-\mathrm{i} \pi / 4} \Delta_{12} & -\Delta_{0} & \Delta_{2} \\ \mathrm{e}^{\mathrm{i} \pi / 4} \Delta_{12} & -\mathrm{e}^{-\mathrm{i} \pi / 4} \Delta_{11} & \Delta_{2} & -\Delta_{0}\end{array}\right]$

The main difference between this and the $C_{2 v}$ Hamiltonian (Eq. 1) are the non-vanishing DE-BE mixing terms. The DE-BE mixing is determined by two matrix elements $\Delta_{11}$ and $\Delta_{12}$, with a phase. The phase reflects the $\pi / 4$ phase associated with the BE eigenstates and the zero phase associated with the DE eigenstates of Eq. 1. Thus, there are two sources for the DE dipole moment. The first is the non-zero $\hat{z}$-polarized dipole moment discussed above, and the second is the in-plane polarized dipole moments resulting from mixing with the BEs. Neither of these effects can be a priori neglected.

The formal structure of the $C_{s}$ Hamiltonian (Eq. 2) resembles the Hamiltonian for a QD in an external magnetic field in the Voigt geometry $\cdot \frac{3}{*}$ One can therefore view the mixing terms as resulting from an effective in-plane magnetic field. An intuitive way of seeing the source for this magnetic field is to describe the hole motion in the magnetic field produced by the spinning electron and vice versa. The spinning electron thus couples the $|\mathrm{DE} 1\rangle$ $(|\mathrm{DE} 2\rangle)$ exciton to the $|\mathrm{BE} 2\rangle(|\mathrm{BE} 1\rangle)$ exciton, resulting in the mixing term $\Delta_{12}$, while the spinning hole couples the $|\mathrm{DE} 1\rangle(|\mathrm{DE} 2\rangle)$ to the $|\mathrm{BE} 1\rangle(|\mathrm{BE} 2\rangle)$ exciton, resulting in the mixing term $\Delta_{11}$. The effective in-plane magnetic field results from the fact that, in the lower symmetry $\mathrm{QD}$, both the electron and the hole have non-vanishing in-plane spin projection expectation values. For the QD in Fig. 2, the facet effectively tilts the QD axis with respect to the crystallographic direction [001]. This "tilt" results in non-zero expectation values for the in-plane spin-components of both the electron and the hole. In contrast, for a QD with $C_{2 v}$ symmetry these expectation values are exactly zero.

Since there is a one-to-one correspondence between the atomistic model basis and the angular momentum basis of Eq. 1. the exchange Hamiltonian of Eq. 2 must have the same formal structure in the angular momentum basis as well. Therefore, we can fit the atomistic model calculated excitonic spectra for obtaining the mixing parameters $\Delta_{11}$ and $\Delta_{12}$ of the simplified $4 \times 4$ Hamiltonian expressed by the angular momentum basis of the symmetric QD as in Eq. 1.

The calculated and fitted values of all the entries in the Hamiltonians (Eq. 1 and Eq. 22) are depicted in Table II. With these entries, the eigenenergies and eigenstates of the four excitons can be straightforwardly calculated. One immediately notes that, in all cases, the symmetric (under electron-hole exchange) BE state couples only to the antisymmetric DE state and the antisymmetric BE state couples only to the symmetric DE state. Thus, the visible DE state has opposite symmetry to the symmetry of the BE state to which it couples (the one polarized along the [110] axis). Indeed, the visible DE state of the QD in Fig. 2 (c) has even symmetry while the $[110$ polarized BE exciton to which it couples has odd symmetry under electron-hole exchange.

The apparent anisotropy between the dipole moments of the two DE eigenstates can be traced to the constructive contribution, due to DE-BE mixing, to the optical activity of one DE eigenstate and destructive contribution to the optical activity of the other eigenstate. If the magnitudes of $\Delta_{11}$ and $\Delta_{12}$ are equal, the destruction is then complete, leading again to only one (like for 
TABLE I. Summary of the calculated values in the presented systems

\begin{tabular}{lcccccl}
\hline & & $\begin{array}{c}\text { Fig. 1 (a) } \\
\text { (flat, symmetric) }\end{array}$ & $\begin{array}{c}\text { Fig. 1 (c) } \\
\text { (tall, symmetric) }\end{array}$ & $\begin{array}{c}\text { Fig. 2 (a) } \\
\text { (flat, truncated) }\end{array}$ & $\begin{array}{c}\text { Fig. 2 (c) } \\
\text { (tall, truncated) }\end{array}$ & \\
\hline$\Delta_{0}$ & $\mu \mathrm{eV}$ & 515 & 315 & 570 & 370 & BE-DE split \\
$\Delta_{1}$ & $\mu \mathrm{eV}$ & 84.7 & 40.7 & 48 & 28 & BE FSS amplitude \\
$\Delta_{2}$ & $\mu \mathrm{eV}$ & 3.88 & -6.51 & -5.0 & -0.5 & DE FSS amplitude \\
\hline$\Delta_{11}$ & $\mu \mathrm{eV}$ & 0 & 0 & 3.71 & 3.27 & BE-DE mixing \\
$\Delta_{12}$ & $\mu \mathrm{eV}$ & 0 & 0 & 0.81 & 12.7 & BE-DE mixing \\
\hline
\end{tabular}

symmetrical QDs) optically active DE eigenstate. Here, however, the visible DE is polarized in-plane.

We carefully note here that the lower-symmetry QDs presented above can be viewed as a proof of concept only. Though clearly most real QDs have lower than $C_{2 v}$ symmetry, a reliable comparison with experiment is impossible without a detailed knowledge of the QD structural shape and its composition and strain distributions. Systematic comparison with experimental measurements must take into account as well the mixed composition $\left(\operatorname{In}_{x} \mathrm{Ga}_{1-x} \mathrm{As}\right)$ of the QD and the resulting lattice disorder. While such studies are beyond the scope of this manuscript, we note that if one assumes a uniform composition profile, the conclusions discussed above remain qualitatively correct for $\operatorname{In}_{x} \mathrm{Ga}_{1-x}$ As disordered systems.

\section{CONCLUSIONS}

We use atomistic model simulations for studying the effect of symmetry reduction on the optical properties of excitons confined in self-assembled InAs/GaAs QDs. We compare between $C_{2 v}$ symmetrical QDs and asymmetrical ones with $C_{s}$ symmetry only. The symmetry in the studied QD is reduced by forming a planar facet between the QD and its host material. We show that, while the symmetry reduction hardly affects the BE eigentates' oscillator strength and polarizations, it strongly affects the DE eigenstates. For symmetric QDs, one DE eigenstate is dark while the other one has a small dipole moment polarized along the QD symmetry axis. For the symmetry "broken" QDs, the DE eigenstates are mixed with the BE eigenstates of opposite symmetry under electronhole exchange. Therefore, they become optically active for in-plane polarized light, like the BEs with which they are mixed. There is very large anisotropy between the dipole moments of the two DE eigenstates, since there are two mixing terms that interfere constructively for one DE eigenstate and destructively for the other one. The calculated excitonic spectrum and its polarization selection rules are in quantitative agreement with recently obtained experimental results.

\section{ACKNOWLEDGEMENTS}

We acknowledge support from the Polish National Science Centre based on decision DEC2011/01/D/ST3/03415. Support from the Israeli Science Foundation, and from the Technion's RBNI is acknowledged as well. Stimulating discussions with G. W. Bryant are acknowledged.
* mzielin@fizyka.umk.pl

1 D. Loss and D. P. DiVincenzo,

Phys. Rev. A 57, 120 (1998)
E. Ivchenko, Y. Fu, and M. Willander, Phys. Solid State 42, 1756 (2000)

3 M. Bayer, G. Ortner, O. Stern, A. Kuther, A. A. Gorbunov, A. Forchel, P. Hawrylak, S. Fafard, K. Hinzer, T. L. Reinecke, S. N. Walck, J. P. Reithmaier, F. Klopf, and F. Schäfer, Phys. Rev. B 65, 195315 (2002).

4 P. Michler, A. Kiraz, C. Becher, W. V. Schoenfeld, P. M. Petroff, L. Zhang, E. Hu, and A. Imamoglu, Science 290, 2282 (2000)

5 C. Santori, M. Pelton, G. Solomon, Y. Dale, and Y. Yamamoto, Phys. Rev. Lett. 86, 1502 (2001).

6 Z. Yuan, B. E. Kardynal, R. M. Stevenson, A. J. Shields, C. J. Lobo, K. Cooper, N. S. Beattie, D. A. Ritchie, and M. Pepper, Science 295, 102 (2002).
7 A. J. Shields, M. P. O'Sullivan, I. Farrer, D. A. Ritchie, R. A. Hogg, M. L. Leadbeater, C. E. Norman, and M. Pepper, Appl. Phys. Lett. 76, 3673 (2000).

8 J. C. Blakesley, P. See, A. J. Shields, B. E. Kardynał, P. Atkinson, I. Farrer, and D. A. Ritchie, Phys. Rev. Lett. 94, 067401 (2005).

9 E. J. Gansen, M. A. Rowe, M. B. Greene, D. Rosenberg, T. E. Harvey, M. Y. Su, R. H. Hadfield, S. W. Nam, and R. P. Mirin, Nat. Photon. 1, 585 (2007).

10 N. Akopian, N. H. Lindner, E. Poem, Y. Berlatzky, J. Avron, D. Gershoni, B. D. Gerardot, and P. M. Petroff, Phys. Rev. Lett. 96, 130501 (2006).

11 R. J. Young, R. M. Stevenson, P. Atkinson, K. Cooper, D. A. Ritchie, and A. J. Shields, New Journal of Physics 8, 29 (2006).

12 M. Muller, S. Bounouar, K. D. Jons, M. Glassl, and P. Michler, Nat. Photon. 8, 224 (2014). 
${ }^{13}$ K. De Greve, L. Yu, P. L. McMahon, J. S. Pelc, C. M. Natarajan, N. Y. Kim, E. Abe, S. Maier, C. Schneider, M. Kamp, S. Hofling, R. H. Hadfield, A. Forchel, M. M. Fejer, and Y. Yamamoto, Nature 491, 421 (2012).

14 W. B. Gao, P. Fallahi, E. Togan, J. Miguel-Sanchez, and A. Imamoglu, Nature 491, 426 (2012).

15 J. R. Schaibley, A. P. Burgers, G. A. McCracken, L.-M. Duan, P. R. Berman, D. G. Steel, A. S. Bracker, D. Gammon, and L. J. Sham, Phys. Rev. Lett. 110, 167401 (2013).

16 E. Poem, Y. Kodriano, C. Tradonsky, N. H. Lindner, B. D. Gerardot, P. M. Petroff, and D. Gershoni, Nat. Phys. 6, 993 (2010).

17 I. Schwartz, E. R. Schmidgall, L. Gantz, D. Cogan, E. Bordo, and D. Gershoni, arXiv (2014), arXiv:1408.2892v1 [quant-ph],

18 E. D. Kim, K. Truex, X. Xu, B. Sun, D. G. Steel, A. S. Bracker, D. Gammon, and L. J. Sham, Phys. Rev. Lett. 104, 167401 (2010).

19 D. Press, K. De Greve, P. L. McMahon, T. D. Ladd, B. Friess, C. Schneider, M. Kamp, S. Hofling, A. Forchel, and Y. Yamamoto, Nat. Photon. 4, 367 (2010).

20 A. J. Ramsay, S. J. Boyle, R. S. Kolodka, J. B. B. Oliveira, J. Skiba-Szymanska, H. Y. Liu, M. Hopkinson, A. M. Fox, and M. S. Skolnick, Phys. Rev. Lett. 100, 197401 (2008).

21 A. Greilich, S. G. Carter, D. Kim, A. S. Bracker, and D. Gammon, Nat. Photon. 5, 702 (2011).

${ }^{22}$ K. De Greve, P. L. McMahon, D. Press, T. D. Ladd, D. Bisping, C. Schneider, M. Kamp, L. Worschech, S. Hofling, A. Forchel, and Y. Yamamoto, Nat. Phys. 7, 872 (2011).

23 T. M. Godden, J. H. Quilter, A. J. Ramsay, Y. Wu, P. Brereton, S. J. Boyle, I. J. Luxmoore, J. Puebla-Nunez, A. M. Fox, and M. S. Skolnick, Phys. Rev. Lett. 108, 017402 (2012).

24 T. Smoleński, T. Kazimierczuk, M. Goryca, T. Jakubczyk, L. Kłopotowski, L. Cywiński, P. Wojnar, A. Golnik, and P. Kossacki, Phys. Rev. B 86, 241305 (2012).

25 M. A. Dupertuis, K. F. Karlsson, D. Y. Oberli, E. Pelucchi, A. Rudra, P. O. Holtz, and E. Kapon,
Phys. Rev. Lett. 107, 127403 (2011).

26 K. F. Karlsson, M. A. Dupertuis, D. Y. Oberli, E. Pelucchi, A. Rudra, P. O. Holtz, and E. Kapon, Phys. Rev. B 81, 161307 (2010).

27 T. Takagahara, Phys. Rev. B 62, 16840 (2000)

28 E. Poem, J. Shemesh, I. Marderfeld, D. Galushko, N. Akopian, D. Gershoni, B. D. Gerardot, A. Badolato, and P. M. Petroff, Phys. Rev. B 76, 235304 (2007).

29 G. W. Bryant, M. Zieliński, N. Malkova, J. Sims, W. Jaskólski, and J. Aizpurua, Phys. Rev. Lett. 105, 067404 (2010).

30 M. Zieliński, M. Korkusiński, and P. Hawrylak, Phys. Rev. B 81, 085301 (2010).

31 M. Korkusinski and P. Hawrylak, Phys. Rev. B 87, 115310 (2013).

32 S. Alon-Braitbart, E. Poem, L. Fradkin, N. Akopian, S. Vilan, E. Lifshitz, E. Ehrenfreund, D. Gershoni, B. D. Gerardot, A. Badolato, and P. Petroff, Physica E 32, 127 (2006)

33 J. G. Tischler, A. S. Bracker, D. Gammon, and D. Park, Phys. Rev. B 66, 081310 (2002).

34 M. Zielinski, J. Phys.: Cond. Matter 25, 465301 (2013).

35 J.-M. Jancu, R. Scholz, F. Beltram, and F. Bassani, Phys. Rev. B 57, 6493 (1998).

36 M. Zieliński, Phys. Rev. B 86, 115424 (2012)

37 P. N. Keating, Phys. Rev. 145, 637 (1966).

38 R. M. Martin, Phys. Rev. B 1, 4005 (1970)

39 A. Beya-Wakata, P.-Y. Prodhomme, and G. Bester, Phys. Rev. B 84, 195207 (2011).

40 W. Sheng, S.-J. Cheng, and P. Hawrylak, Phys. Rev. B 71, 035316 (2005).

41 G. Bester and A. Zunger, Phys. Rev. B 71, 045318 (2005)

${ }^{42}$ R. Singh and G. Bester, Phys. Rev. Lett. 104, 196803 (2010).

43 M. Zieliński, Phys. Rev. B 88, 155319 (2013)

44 P. Michler, Single Quantum Dots: Fundamentals, Applications and New Concepts, Physics and Astronomy Online Library (Springer, New York, 2003).

45 B. J. Spencer and J. Tersoff, Phys. Rev. B 87, 161301 (2013). 